Paper ID \#19802

\title{
The NECST Program - Networking and Engaging in Computer Science and Information Technology Program
}

\section{Dr. Katherine G. Herbert, Montclair State University}

Katherine G. Herbert, Ph.D. Associate Professor of Computer Science, Montclair State University.

Dr. Katherine Herbert is an Associate Professor of Computer Science at Montclair State University.

Dr. Herbert currently researches database management, data warehousing and data quality issues in biological, biochemical and sustainability-related data sets. Dr. Herbert received her Ph.D. in Computer Science in 2004 from the New Jersey Institute of Technology for studying data quality issues in biological databases. Since completing her Ph.D., she has continued to publish Dr. Herbert's primary research is in biological databases with a specialty in phylogenetic databases. Presently, she is examining the data integration problems with regards to integrating protein-ligand data with respect to the phylogenetics relationships within the protein data with Dr. Nina Goodey from the Department of Chemistry and Biochemistry at MSU. This work is has been funded by the Montclair State University Sokol Institute for Pharmaceutical Life sciences where she is developing a prototype system and been publish in ACM SIGMOD, ISMB CSHALS and the Faculty of 1000 blog Naturally Selected. Dr. Herbert is also a recently looking at problems regarding sustainability data and mobile applications. This work with Dr. Emily Hill, Dr. Jerry Fails and Dr. Jennifer Bragger, has been funded by the PSEG Institute for Sustainability Sciences. This work has been published in IEEE Big Data and ACM CSCW. Dr. Herbert is also the Principle Investigator for the National Science Foundation funded S-STEM Networking and Engaging in Computer Science and Information Technology (NECST) Program here at Montclair State University (NSF award 1259758). The NECST Program funds students, regardless of background, who are interested in pursuing a graduate degree in computer science. Students from computing related fields as well as other fields are welcome to apply. These students are then mentored through a network of mentors from not only MSU, but also neighboring universities who participate in NECST. Through her work with the Science Informatics Program, Dr. Herbert has also published a number of Computer Science and Interdisciplinary education papers in venues such as DIMACS, CUR and ACM Special Interest Group in Computer Science Education (SIGCSE).

\section{Dr. Thomas J Marlowe, Seton Hall University}

Thomas Marlowe is Professor of Mathematics and Computer Science at Seton Hall University, where he has taught for the past 37 years, and holds Ph.D.s in both disciplines from Rutgers University. He has broad research interests in computer science, having published on programming languages and compiler optimization, real-time systems, software engineering and collaborative development, algorithms, and computer science pedagogy. He is co-PI on the NECST grant.

\section{Dr. Jerry Alan Fails, Boise State University}

Dr. Jerry Alan Fails is an associate professor in the Computer Science Department at Boise State University in Boise, Idaho. His primary area of research is Human-Computer Interaction, with a focus on technologies that support children's creativity, mobility, and collaboration and promote activity and exploration of the world around them. He has mentored several graduate and undergraduate students and is deeply invested in engaging students in research experiences and providing students with the tools they need to succeed after graduating.

\section{Dr. Cyril S Ku, William Paterson University}

Dr. Cyril S. Ku received the B. S. degree in Computer Science from Utah State University, Logan, Utah, USA, in 1980, the M. S. degree in Computer Science and Applications from Virginia Polytechnic Institute and State University, Blacksburg, Virginia, USA, in 1982, and the Ph. D. degree in Computer Science from Northwestern University, Evanston, Illinois, USA, in 1989.

From 1982 to 1985, he was a Software Engineer in the field of traffic and transportation engineering. He conducted application research in software engineering, databases, and data science as Member of 
Technical Staff and as Systems Analyst at Bell Communications Research (Bellcore, now Ericsson, 19891996), as Senior Technical Staff Member at AT\&T (1996-2000), and as Senior Information Technology Specialist at IBM (2000-2002).

He has been a Full Professor at William Paterson University, Wayne, New Jersey, USA since 2014 (Assistant Professor: 2002-2009, Department Chair: 2007-2013, Associate Professor: 2009-2014). He received an Excellence Award for Faculty Service in 2009. His research interests include software engineering, databases, data warehouses (big data), data mining, and automated reasoning and theorem proving. He is a member of ACM (Association for Computing Machinery), IEEE (Institute of Electrical and Electronics Engineers) Computer Society, and UPE (Upsilon Pi Epsilon).

\section{Dr. Kelly M Goedert, Seton Hall University Dr. Emily Hill, Drew University}

Emily Hill is Assistant Professor of Computer Science at Drew University, a small liberal arts college outside of New York City. Her research interests involve creating intuitive software engineering tools for novice developers by utilizing the natural language information present in source code. She is also passionate about undergraduate computer science education.

\section{Dr. Nina M Goodey, Montclair State University}

Dr. Nina Goodey is an associate professor in the Department of Chemistry and Biochemistry at Montclair State University in Montclair, New Jersey, U.S.A. She has expertise in enzymology and is interested in engineering of soil enzymatic function. She enjoys mentoring student research.

\section{Fr. Donal Thomas MacVeigh S.J.}




\section{The NECST Program - Networking and Engaging in Computer Science and Information Technology Program}

\section{Introduction}

In this paper, we describe the NECST Program and its innovative mentorship structure for transitioning graduate students in computer science whose undergraduate experiences may be in other disciplines. NECST employs several activities that provide the additional scaffolding to support students as they make this transition. While we believe these activities may be suited for other situations, the program helps address the unique challenges northern New Jersey faces with relation to graduate studies in computing fields.

There have been significant efforts toward addressing the current and future shortfalls and mismatches in the computing, information, and technology workforce [1]. These efforts include attracting more students into computer science, fostering a realistic and interdisciplinary approach to computing, and increasing cooperation and collaboration between institutions. The NECST Program [2], funded through the NSF S-STEM program [3], tackles these challenges in a novel way. Most approaches to increasing STEM (science, technology, engineering and mathematics) enrollment in general - and computer science enrollment in particular - focus on secondary or even primary school students or underclass college students. NECST, however, looks to interest undergraduates close to graduation, recent alumni, or returning students from any discipline in graduate study in computer science. These interests are motivated as a change in career direction, or equally often as a means to acquire skills, concepts, and frameworks that complement their original discipline or career plans [4]. This can be an advantage for students of all disciplines, including undergraduate computing majors, since employers have remarked that many computer science majors, although possessing basic knowledge and dedication, lack understanding and depth, critical thinking, and communication skills [5].

NECST (Networking and Engaging in Computer Science and Information Technology) is supported by a consortium of (currently four) institutions in northern New Jersey. The region hosts numerous higher education institutions, public and private, and is characterized by a high demand for computing and information technology. Through the program, students receive a two-year scholarship to pursue a practice-oriented, career-centered Master's degree in Computer Science at Montclair State University. The other three institutions, Saint Peter's University, Seton Hall University and William Paterson University, have well-established undergraduate computer science programs but currently no graduate program.

The scholarship is not restricted to those with undergraduate majors in computer science or related fields, or even to STEM majors; in fact, its greatest benefit is arguably to those with an interest in computing or technology but have a very different undergraduate background. Students benefit from a structured but flexible framework of mentoring and group activities, including student-organized group support.

The program has had two welcomed consequences for the partner institutions. It has fostered increased cooperation on opportunities for collaboration among the computer science programs at 
the institutions involved as well as between the computer science departments and the diverse departments from which students have applied. In this poster, we introduce the NECST program, address its structure and administration. and discuss its preliminary results and future directions.

Northern New Jersey is one of the most densely populated areas of the nation, rich with history and diversity. Often, New Jersey becomes one of the first areas many immigrant groups settle when they first come to the country. Due to New Jersey's history, many colleges and universities have sprung up, servicing the different populaces in the state. The State of New Jersey recognizes 56 separate higher education institutions, 32 of which offer baccalaureate level or higher education. Twenty-nine of these have Bachelor's degrees in computer science and ten offer Master's degrees or higher. On the other hand, New Jersey is a known "sending” state, with more students leaving the state for college than coming in from elsewhere [6]; this remains true for graduate education.

With so many institutions servicing a diverse populace in a small geographic area, students are presented with many options for study. Many elect undergraduate majors without fully understanding the nature of their chosen field or its career potential. Conversely, many initially shy away from STEM, and especially from computer science, perhaps due to mistaken ideas about the field and its practitioners, possibly in combination with dread of the technical requirements of the discipline. The common misconception that all computer scientists live in a windowless box, coding all day in isolation is unattractive to many students.

The difficulty for many students is that by the time they have a more realistic view of their own and other disciplines and their career potential, changing to a computer science major may require an additional year or even two of undergraduate study, and combining computer science with their current major may take even longer. Considering graduate education, even for students in STEM fields at smaller institutions, the pathway to advanced studies can seem unclear. Moreover, with so many schools in the region, expertise and resources for sub-specializations in computer science, and opportunities for interdisciplinary study, tend to be dispersed among the schools. Therefore, students also have the challenge of trying to select schools that may only meet some of the students' learning objectives. For non-STEM students, and to some extent for STEM students changing fields, the problem is even worse. Pursuing a computer science degree without a clear welcome and without significant support seems risky, and many will be reluctant to attempt it when they should be attempting to establish their lives and careers.

It is well-documented and a serious concern that the United States is graduating too few students in STEM, particularly in the computing and information sciences and data sciences, and that graduates with this background are needed in a wide range of fields, including finance, science, psychology and cognitive science, and communication [7, 8, 9, 10, 11, 12]. Demand and compensation both in the computing, information, and data sciences, and in these interdisciplinary areas as well, is very high, with opportunities for promotion, growth and interesting career opportunities and intellectual challenges.

\section{The NECST program}

This combination of demand, risk, and opportunity argues strongly for a program that can attract and 
support students from a variety of undergraduate majors in graduate study in computer science, and northern New Jersey is an ideal location in which to locate such a program. In response to this, we have created the NECST Program: Networking and Engaging in Computer Science and Information Technology in Northern New Jersey. This program, funded by an NSF S-STEM grant, has formed a collaboration between Montclair State University (MSU), Saint Peter's University (SPU), Seton Hall University (SHU), and William Paterson University (WPU) to help form a structure for students to interact with faculty from all members of this collaboration and receive a $\$ 10,000$ scholarship to pursue a Master's of Science in Computer Science (MSCS) degree at Montclair State University. The program supports MSU, SPU, SHU and WPU to formalize their collaboration, outreach to other schools to join the collaboration and create avenues to harness each other's resources so that the computing communities at these member institutions can benefit from each other. Although other institutions reach out to non-CS and even non-STEM majors (see for example [5]), the NECST program appears to be unique in providing the combination of financial support and mentoring together with regional inter-institutional collaboration.

\section{Program administration}

This degree is a 33-credit program. There are three three-credit required courses, plus seven elective courses for a total of 21 credits, and one three-credit culminating experience, a Master's Thesis or Master's Project. The MSCS program expects students to have prior background, including programming in a high-level language, plus knowledge of computer architecture (or a related hardware field), data structures and algorithms, discrete mathematics, and precalculus. For students without this background, the department requires the student to complete a prerequisite or "bridge" core-knowledge program covering in any missing areas. This table provides students with an attainable path to the MSCS degree in a two-year period if they satisfy the prerequisite structure, and three years if they need to complete the "Core Knowledge” program. For students interested in an interdisciplinary degree, students may choose a computer science faculty member as a mentor, and then may select up to four electives (12 credits) from other academic departments with the permission of the mentor, the graduate program coordinator the CS Department, and the graduate program coordinator of the department hosting the course. The MSCS program has three options for study, all with the same Core Knowledge Program and admissions requirements. Students can also elect to take an Information Technology (IT) Concentration or an Applied Information Technology (AIT) Concentration. The AIT program has Professional Science Master's affiliation. Information about electives and all programs can be found at http://www.cs.montclair.edu/academics/graduate/graduate.html.

The NECST Program is currently funded through the 2017 academic year. Each year of the program, we have admitted 6 to 7 new students, with an expected 26 students to complete the program in the five-year program. Students are eligible for the program if they meet the following criteria: 3.0 GPA in Pre-Calculus or a similar measure of potential in mathematics; US citizens, nationals, admitted refugees or permanent residents; enrolled or enrolling fulltime in an MSU Department of Computer Science Master's program; and financial aid eligible (as determined by Office of Financial Aid) [3].

As a part of our degree program, we can accommodate students with our Pre-requisite program if they have completed Pre-Calculus or a similar upper level mathematics course. This policy is a part 
of MSU Department of Computer Science Graduate Program general graduate admissions policies. MSU has the Red Hawk Math Learning Center, where we can have a student's skills evaluated to determine if their skills place them at or above Pre-Calculus. Students with a course other than PreCalculus would need to be tested by the Red Hawk Math Readiness Center. Excellent students, especially those in Non-CS areas, will be advised if math potential is below the expected criteria.

Along with our requirements, we have also identified a number of indicators that help in assessing students' probable success in our program. For example, if students complete any of the following, we would see these activities as positive indicators: successful completion of activities like undergraduate research (in any field), internships, campus activities, and/or appropriate professional work experience. In addition to these requirements, as per the project description, one recipient of each cohort year will be from each of the collaborating institutions, if there is an eligible applicant. The other three to four places in the cohort class are for students from any background and any institution.

Besides enabling students to obtain the MSCS degree full-time, the project team has also identified mentoring and creation of services as key to this program collaboration. Therefore, we have supporting activities to help with recruitment, retention and monitoring of student progress including inter-institutional faculty mentoring, career support activities and mentorship styles designed for each student's needs.

Faculty at MSU mentor all students in the program and students have the choice of working with faculty partners from other institutions. For students from SPU and SHU, once students have selfidentified as candidates, they will work with a faculty member there, coupled with a mentor from MSU to transition into their graduate studies at MSU. If students demonstrate interest earlier than their senior year, a faculty member at that institution will advise them as they select their computer science and other courses to facilitate the transition or to increase the value of the MSCS to their career plans. Students accepted into this program who come from a non-NECST consortium member will be assigned an MSU advisor; such students as well as MSU undergraduates accepted as NECST scholars, will interact with the SPU and SHU faculty through other activities.

Mentoring exercises are delivered in a flexible manner so that the student-mentor teams can choose the best mechanism(s) for communication —on campus, email and phone, distance-based tools, or social media, all of which are supported by the participating institutions where these resources are not freely available. All three institutions are technology-intensive and support faculty in distance and on-line education and in using other tools.

\section{Preliminary results}

The project has provided mentorship and support services, optionally placing students in degree- and career-relevant internships. The PI and several Co-PIs have traveled to other participating institutions to conduct outreach, including presentations and meetings with prospective students. In the first three years, a total of 22 students have received \$10,000 per /year scholarships-15 (68\%) students from other undergraduate majors, 11 (50\%) from consortium institutions, and 10 (45\%) from underrepresented groups. 6 (27\%) will have received master's in science (MS) degrees by 
August 2016, 10 (54\%) are still pursuing graduate study, and 6 (27\%) have left the program for a combination of academic and personal reasons. Successful students have come from mathematics, philosophy, biology, psychology, Asian studies and other areas. Anecdotally, the presence of students from diverse undergraduate backgrounds has made for better quality teams and arguably better quality classes (compare [13, 14]). Every student graduating from the program has been employed in their chosen career within six months of graduation. While several students have left the program for full-time employment in computing, continuing their graduate studies part-time, these should be viewed as program successes. Finally, student feedback has been entirely positive, and all students in the first cohort have seen improvement in the opportunities for which they have been eligible, including internship, employment, and research opportunities.

By including four universities with very different missions, serving similar but different populations, we are seeing often underrepresented minority groups more strongly represented. In the first two cohorts, totaling 12 students, have four students with an African-American background, one with a Hispanic background, and two women.

Additionally, the success of NECST inspired MSU's Department of Chemistry and Biochemistry to adapt this model to their curricular needs and create the Opening Pathways, Engaging, and Networking in Chemistry in Northern New Jersey (OPEN-NJ) program. The program has also created synergistic connections with our colleague-departments at all of our universities. At SHU, the Department of Psychology has seen this opportunity for their students as a way to help mentor the students into alternative career paths and also strengthen student skills for data visualization and analysis.

\section{Conclusion and future directions}

The NECST program is a unique program that supports students' post-baccalaureate objectives in computer science. It facilitates a late transition into computer science, not as part of the undergraduate experience, but into a practice-oriented Master's degree program, and not just for other STEM majors, but for non-STEM students as well, providing not just scholarships but intense mentoring and gateways to internship opportunities. It is also a regional collaboration that creates both interdisciplinary and inter-institutional links and shared activities. The program can be readily replicated, given the proper elements including funding, institutions willing to collaborate, and dedicated faculty members. The program has attracted a student population with academic and social diversity, and provides flexibility and initiative in incorporating student backgrounds and career plans in a graduate plan of study.

In our future plans, we hope to broaden this program to include all STEM graduate programs at MSU, to reach out to additional institutional partners in northern New Jersey, and to improve outreach and dissemination. We aim to increase joint activities, and collaborate with other related interdisciplinary programs and groups, such as the DAVA program at SHU and the Seton Hall/William Paterson University group on data visualization. Another forthcoming project is to encourage NECST graduates as alumni mentors for the entering cohort. Additionally, the program intends to approach industry partners to seek dedicated internships or perhaps funding of additional scholars attached to NECST's successor program. 
With all of our positive outcomes, there remain some challenges. The northern New Jersey region notoriously has one of the highest costs of living in the country. While the support is sizable, many students still have challenges with supporting themselves and maintaining full-time status. The program will be more effective if schools can identify interested students earlier, to inform and advise them of the opportunity and ways to make it easier and more valuable. NECST faculty at each institution are working within their own departments and with other departments to attempt to do so. Moreover, with more experience, additional assessments can be developed and administered. Finally, if the program obtains additional funding to extend beyond the life of the initial grant, it will be necessary to retain faculty and administration interest and support, and to handle changeovers in personnel. In summary, the NECST program has already shown an approach to attracting more students into computing, and helping them succeed. We plan to encourage more students to consider graduate STEM education by providing limited funding for upper-class undergraduates to explore this opportunity. Finally, the proposed project will include significant industry involvement, including an advisory board, networking with industry, and facilitating the arrangement of internship and part-time job opportunities and support, so that students can work while continuing to pursue full-time study.

\section{Acknowledgments}

This work is supported by NSF S-STEM Award 1259758. We would like to acknowledge the Administration, Staff and Faculty at MSU, SHU and SPU for their support, and would also like to thank James Benham, Angel Gutierrez and the Department of Computer Science at MSU, the Department of Mathematics \& Computer Science at SHU, the Department of Computer Science at SPU, the Department of Computer Science at WPU, and Susan Nolan and the Department of Psychology at SHU for participating in this program. We would also like to recognize the New Jersey Academy of Science for supporting our meetings. Finally, we would like to thank all of the students who have shown interest in NECST.

\section{References}

[1] Bidwell, A., Tech Companies Work To Combat Computer Science Education Gap. US News \& World Report, December 27, 2013. Available at http://www.usnews.com/news/articles/2013/12/27/tech-companies-work-to-combat-computerscience-education-gap

[2] NECST Project, NECST Website, http://www.cs.montclair.edu/ necst/

[3] National Science Foundation, S-STEM Request for Proposals, http://www.nsf.gov/pubs/2012/nsf12529/nsf12529.htm

[4] Northern Illinois University, Seeking a Second Degree, after an Undergraduate Degree in a Field Other Than Computer Science, http://www.cs.niu.edu/graduate/nonCSGrad.shtml. Accessed 9/4/2014.

[5] Spark, D., 2014. Trying to Undo the Skills Mismatch Problem. The Dice Report, September 13, 2012.

[6] Diane D’Amico, 2012. Going Away to School: New Jersey Loses 40 percent of four-year college students, Press of Atlantic City, December 2, 2011, http://www.pressofatlanticcity.com/news/press/new_jersey/going-away-to-school-new-jerseyloses-percent-of-four/article_381c2ba2-1c7f-11e1-9025-001871e3ce6c.html, last accessed 
August 11, 2012.

[7] Bureau of Labor Statistics, 2012. http://www.bls.gov/ro2/, last accessed August 13, 2012.

[8] Computer Research Associates, The Taulbee Survey, http://cra.org/resources/taulbee/, accessed September 2014.

[9] Dwoskin, E., 2014. Big Data's High-Priests of Algorithms: 'Data Scientists' Meld Statistics and Software for Find Lucrative High-Tech Jobs. Wall Street Journal Tech Blog, August 8, 2014. http://online.wsj.com/articles/academic-researchers-find-lucrative-work-as-big-data-scientists1407543088?mod=WSJ_hp_LEFTWhatsNewsCollection

[10] FlipDog, Computer Science Finance Jobs, http://www.flipdog.com/results.php?k=Finance\&l=Computer+Science\&x=0\&y=0.

[11] Newark Star Ledger, Want College to Pay Off, October 18, 2016, p 1 \& 6.

[12] President's Council of Advisors on Science and Technology, Report to the President: Engage to Excel: Producing One Million Additional College Graduates with Degrees In Science, Technology, Engineering, and Mathematics, February 2012.

[13] Astrachan, O., Osborne, R. B., Lee, I., Beth, B., and Gray, J., 2014. Diverse learners, diverse courses, diverse projects: learning from challenges in new directions. In Proceedings of the 45th Annual SIGCSE Conference on Computer Science Education. 177-178.

[14] Goldberg, D. S. and White, E. K., 2014. E pluribus, plurima: the synergy of interdisciplinary class groups, 457-462, In Proceedings of the 45th Annual SIGCSE Conference on Computer Science Education. 457-462. 